\title{
Nonsingular rings with a countable-dimensional annihilator base
}

\author{
By John Hannah and K. C. O'Meara \\ (Received December 20, 1983)
}

\section{Introduction}

If a nonsingular ring $R$ happens to be a countable-dimensional algebra over some field, then, as shown in [5] and [4], the structure of the maximal right quotient ring $Q_{\max }(R)$ is surprisingly restricted. No extensions of these results seem to be known if $R$ is countable-dimensional over just a subdivision ring or even over a non-central subfield. Here we consider an even weaker condition, namely that some nonzero left annihilator ideal $J$ of $R$ has a countable-dimensional annihilator base (CDAB). This is satisfied, for example, if for some division ring $K \subseteq R$ there is a countable-dimensional vector space ${ }_{K} V \subseteq{ }_{K} J$ such that $V$ intersects non-trivially with all left annihilator ideals $J^{\prime} \subseteq J$ (§2 contains the precise definition of a CDAB). Our principal result (Theorem 2) states that then either $Q_{\max }(R)$ has a nonzero Type $I$ part or $R$ cannot be a right Utumi ring. Right Utumi rings share (by definition) an important property of right self-injective rings - their complement right ideals are right annihilator ideals.

The other main theorem, and on which the principal result hinges, is that if $f$ is an idempotent of a regular, right self-injective ring $Q$, and $Q f$ has a $\mathrm{CDAB}$, then $f Q$ must be of Type $I_{f}$ (Theorem 1 ).

One surprising corollary of these theorems is that if $R$ is a prime, right Utumi ring with a nonzero right ideal which is of countable dimension as a left vector space over some division ring $K \subseteq R$, then $R$ must be right Goldie (Corollary 4). This ties in with J. Lawrence's result [6] that a countable-dimensional self-injective algebra is Artinian. It is also consistent with a more general theme that a nonsingular irreducible ring $R$ which satisfies a countability condition either satisfies a finiteness condition or is, in some sense, a long way from being self-injective.

\section{Definitions, Notation, and Background}

Rings are associative with identity. The left annihilator of a set $X$ in a ring $R$ is denoted as $l_{R}(X)$ or $l(X)$ depending on the context. Similarly 
$r_{R}(X)$ or $r(X)$ denotes the right annihilator.

For a module $A$ (over a general ring), $E(A)$ denotes its injective hull. For a natural number $n, n A$ denotes a direct sum of $n$ copies of $A$. We write $A \leqslant B$ to indicate a module $A$ is subisomorphic to a module $B$, and $A \leqslant_{e} B$ to indicate the submodule $A$ is essential in the module $B$.

For the general background on nonsingular rings and maximal right quotient rings of such rings, as well as uniform modules and the uniform dimension of a finite-dimensional module, see Goodearl [2]. We denote the maximal right quotient ring of a right nonsingular $\operatorname{ring} R$ by $Q_{\max }(R)$. For the theory of regular, right self-injective rings $Q$, and the associated theory of types, see Goodearl [3]. We remind the reader that $Q$ has a nonzero Type $I$ part exactly when it contains a nonzero abelian idempotent $e$ (all idempotents in $e Q e$ are central in $e Q e)$. Also a nonsingular injective module $M_{R}$ is of Type $I_{f}$ exactly when $M$ is directly finite and each nonzero submodule of $M$ contains a nonzero abelian submodule.

A right Utumi ring is a right nonsingular ring in which each complement right ideal is a right annihilator ideal, equivalently, every right ideal of $R$ with zero left annihilator is essential in $R$. These rings were introduced by Utumi in [7]. In terms of $Q=Q_{\max }(R)$, a right nonsingular ring $R$ is right Utumi if and only if $Q$ is left intrinsic over $R$, that is nonzero left ideals of $Q$ intersect nontrivially with $R$ [7, Theorem 2.2]. In particular, regular right self-injective rings, semi-prime right and left Goldie rings, commutative semi-prime rings, and the nonsingular right $C S$-rings of [1] are all right Utumi rings.

Let us say that a module ${ }_{K} V$ has countable uniform dimension if $V$ contains an essential submodule which is a countable direct sum of uniform $K$-modules, equivalently $V$ contains only countable direct sums of nonzero submodules and each nonzero submodule contains a uniform submodule. For example this is true if ${ }_{K} V$ is a countable-dimensional vector space over a division ring $K$, or if ${ }_{K} V$ is a countably generated unitary module over a semisimple Artinian ring $K$, or even if ${ }_{K} V$ is a countably generated nonsingular module over a left nonsingular ring $K$ whose maximal left quotient ring has a countably generated essential left socle.

Finally we introduce a new concept :

Definition. A left annihilator ideal $J$ of a ring $R$ has a countabledimensional annihilator base (CDAB) if there is a subring $K$ of $R$ and a $K$-module ${ }_{K} V \subseteq{ }_{K} J$ such that ${ }_{K} V$ has countable uniform dimension and for all left annihilator ideals $0 \neq J^{\prime} \subseteq J$,

$$
J^{\prime} \cap V \neq 0 .
$$


Note that there is no loss in generality in assuming that ${ }_{K} V$ is actually a (countable) direct sum of uniforms.

\section{The Main Theorems}

THEOREM 1. Let $Q$ be a regular, right self-injective ring and suppose $f$ is an idempotent such that $Q f$ has a countable-dimensional annihilator base. Then $f Q$ is of Type $I_{f}$.

Proof. Let ${ }_{K} V$ be a $\mathrm{CDAB}$ for $Q f$, for some subring $K$ of $Q$. Let

$$
V_{1} \subset V_{2} \subset \cdots \subset V_{n} \subset \cdots
$$

be a chain of $K$-submodules of $V$ such that each $V_{n}$ has uniform dimension $n$ and $\bigcup_{1}^{\infty} V_{i}$ is an essential submodule of ${ }_{K} V$.

Firstly we observe that $f Q$ must be directly finite, otherwise by [3, Theorem 10.19 and Proposition 10.21] $Q f$ contains an uncountable direct sum $\underset{\alpha}{\oplus} Q a_{\alpha}$ of nonzero left ideals and then $\sum_{\alpha} Q a_{\alpha} \cap V$ is an uncountable direct sum of nonzero $K$-submodules of $V$, contrary to the assumption that ${ }_{K} V$ has countable uniform dimension.

Let $f_{1} Q$ and $f_{2} Q$ be respectively the Type $I_{f}$ and Type $I I_{f}$ parts of $f Q$, where $f_{1}, f_{2}$ are idempotents in $f Q f$. Then $f Q=f_{1} Q \oplus f_{2} Q$, and $Q f_{2}$ inherits a CDAB from $Q f$, namely $V \cap Q f_{2}$. Thus in order to show $f Q$ is of Type $I_{f}$ it will suffice to assume $f Q$ is of Type $I I_{f}$ and then deduce that $f Q=0$.

So we assume $f Q$ is of Type $I I_{f}$ and has ${ }_{K} V$ as a CDAB. Since the Type $I$ part of $f Q$ is zero, by [3, Proposition 10.28 ] each submodule of $f Q$ can be written as a direct sum of 3 pairwise isomorphic submodules. (The choice of a 3 -part splitting is inspired by the observation $\sum_{1}^{\infty} 1 / 3^{n}=1 / 2$.) We use this property to inductively construct independent summands $B_{1}, \cdots, B_{n}, \cdots$ of $f Q$ such that

(1) $3 B_{n} \cong B_{n-1} \quad(\forall n>1)$

(2) $l\left(B_{1}+\cdots+B_{n}\right) \cap V_{n}=0$

(3) $f Q=\left(B_{1} \oplus \cdots \oplus B_{n}\right) \oplus C_{n}$ for some $C_{n} \cong\left(B_{1} \oplus \cdots \oplus B_{n}\right) \oplus B_{n}$.

To begin the induction, we write $f Q=A_{1} \oplus A_{2} \oplus A_{3}$ where $A_{1} \cong A_{2} \cong A_{3}$. Since $\bigcap_{1}^{3} l\left(A_{i}\right) \cap Q f=0$, we have $\bigcap_{1}^{3}\left(l\left(A_{i}\right) \cap V_{1}\right)=0$. But each $l\left(A_{i}\right) \cap V_{1}$ is a $K$-submodule of the uniform module $V_{1}$, so we can choose $B_{1} \in\left\{A_{1}, A_{2}, A_{3}\right\}$ such that

$$
l\left(B_{1}\right) \cap V_{1}=0 .
$$

For $C_{1}$ we take the sum of the two $A_{i}$ not equal to $B_{1}$. Clearly (2) and 
(3) hold.

Now suppose for some $n \geqslant 1$ we have constructed $B_{1}, \cdots, B_{n}$ with the desired properties (1), (2), (3). Noting that $B_{i} \cong 3^{n-i} B_{n}$ for $i=1, \cdots, n$ by (1), and that $C_{n} \cong\left(B_{1} \oplus \cdots \oplus B_{n}\right) \oplus B_{n}$ by (3), we can obtain a decomposition of $C_{n}$ as a direct sum of $\left(3^{n-1}+3^{n-2}+\cdots+3+1\right)+1=\left(3^{n}+1\right) / 2$ copies of $B_{n}$. Splitting each of these summands into a direct sum of 3 pairwise isomorphic modules then produces a decomposition

$$
C_{n}=D_{1} \oplus \cdots \oplus D_{k}
$$

where $k=3\left(3^{n}+1\right) / 2, D_{1} \cong D_{2} \cong \cdots \cong D_{k}$, and $3 D_{i} \cong B_{n}$ for each $i$. Let $Y=$ $l\left(B_{1}+\cdots+B_{n}\right) \cap V_{n+1}$, and note that $Y$ is a $K$-submodule of $V$. From (2) we have

$$
V_{n} \oplus Y \subseteq V_{n+1}
$$

and so since $V_{n}$ and $V_{n+1}$ have uniform dimensions of $n$ and $n+1$ respectively, either $Y=0$ or $Y$ is a uniform submodule of ${ }_{k} V$. From (3), $B_{1}+\cdots+$ $B_{n}+D_{1}+\cdots+D_{k}=f Q$ implies $\bigcap_{i=1}^{k} l\left(B_{1}+\cdots+B_{n}+D_{i}\right) \cap Q f=0$. Hence

$$
\bigcap_{i=1}^{k}\left(l\left(B_{1}+\cdots+B_{n}+D_{i}\right) \cap V_{n+1}\right)=0 .
$$

But each $l\left(B_{1}+\cdots+B_{n}+D_{i}\right) \cap V_{n+1}$ is a $K$-submodule of $Y$, and $Y=0$ or is uniform. Thus for some $j$

$$
l\left(B_{1}+\cdots+B_{n}+D_{j}\right) \cap V_{n+1}=0 .
$$

Set $B_{n+1}=D_{j}$ and $C_{n+1}=$ the sum of all the $k-1$ other $D_{i}$.

From $3 D_{j} \cong B_{n}$ we have $3 B_{n+1} \cong B_{n}$, giving (1). Clearly (2) holds for $n+1$. Also $C_{n}=B_{n+1} \oplus C_{n+1}$ so

$$
f Q=B_{1} \oplus \cdots \oplus B_{n} \oplus C_{n}=\left(B_{1} \oplus \cdots \oplus B_{n+1}\right) \oplus C_{n+1}^{\prime}
$$

and

$$
\begin{aligned}
C_{n+1} & \left.\cong(k-1) B_{n+1} \quad \text { (since each } D_{i} \cong B_{n+1}\right) \\
& =\left(\left(3^{n}+3^{n-1}+\cdots+3+1\right)+1\right) B_{n+1} \\
& \cong\left(B_{1} \oplus \cdots \oplus B_{n} \oplus B_{n+1}\right) \oplus B_{n+1}
\end{aligned}
$$

(since $B_{i} \cong 3^{n-i} B_{n} \cong 3^{n+1-i} B_{n+1}$ )

which establishes (3). This completes the induction.

Let $B=E\left(B_{1} \oplus B_{2} \oplus \cdots\right) \subseteq f Q$. By property $(2), \quad l(B) \cap V=0$. In consequence, $l(B) \cap Q f=0$ because $V$ is a $\mathrm{CDAB}$ for $Q f$. Hence $B=f Q$. On the other hand by property $(3), 2\left(B_{1} \oplus \cdots \oplus B_{n}\right) \leqslant f Q$ for all $n$, whence $2 B \leqslant$ 
$f Q$ by [3, Proposition 9.22] since $f Q$ is directly finite. Thus $2(f Q) \leqslant f Q$. The direct finiteness of $f Q$ now forces $f Q=0$, as required.

The above proof is based on an outline given to us by K. R. Goodearl, after he saw our original (much longer) proof.

THEOREM 2. Let $R$ be a right nonsingular ring which which has a nonzero left annihilator ideal with a countable-dimensional annihilator base. Then either the Type I part of $Q_{\max }(R)$ is nonzero or $R$ is not a right Utumi ring.

Proof. Let $Q=Q_{\max }(R)$ and suppose $R$ is right Utumi. Let $J$ be a nonzero left annihilator ideal of $R$ with a CDAB, say ${ }_{K} V$ for some subring $K \subseteq R$. Then $J=Q f \cap R$ for some $f=f^{2} \in Q$. Now since $R$ is right Utumi, $Q$ is left intrinsic over $R$ by [7, Theorem 2.2] and it follows that ${ }_{K} V$ is also a $\mathrm{CDAB}$ for $Q f$ in the ring $Q$. By Theorem 1 we conclude that $f Q$ is of Type $I_{f}$ and so $Q$ has a nonzero Type $I$ part.

\section{Corollaries}

Although Corollaries 1, 3 and 4 (below) are stated in a form which relies on Theorems 1 and 2 only in the case where a CDAB is a countable dimensional vector space over some division ring $K \subseteq R$ (this case seems the most interesting), these corollaries remain valid when the ideals in question have countable uniform dimension over an arbitrary subring $K$ of $R$.

COROllaRy 1. Suppose $R$ is a right Utumi ring with a nonzero left ideal of countable dimension as a left vector space over some division ring $K \subseteq R$. Then $Q_{\max }(R)$ has a nonzero Type I part.

REMARK. For $R$ meet-irreducible (two-sided ideals intersect nontrivially), this means $R$ has uniform right ideals.

Proof. Let $V=R a \neq 0$ be a principal left ideal of $R$ with $\operatorname{dim}_{K} V$ countable. Let $J=l(r(V))$.

Claim: ${ }_{k} V$ is a $\mathrm{CDAB}$ for the left annihilator ideal $J$ of $R$.

For let $0 \neq J^{\prime} \subseteq J$ be a left annihilator ideal of $R$. We wish to show $J^{\prime} \cap V \neq 0$. Let $Q=Q_{\max }(R)$ and write $Q a=Q f, a Q=e Q$, where $e, f$ are idempotents in $Q$. Observe that $J=l_{R}\left(r_{R}(Q f)\right)=l_{R}((1-f) Q \cap R)=Q f \cap R$. If we let $r_{Q}\left(J^{\prime}\right)=(1-g) Q$ for $g=g^{2} \in Q$, then $J^{\prime}=l_{R}\left(r_{R}\left(J^{\prime}\right)\right)=l_{R}((1-g) Q \cap R)$ $=Q g \cap R$. Also $0 \neq Q g \cap R=J^{\prime} \subseteq J \subseteq Q f=Q a$, so $0 \neq y a \in Q g$ for some $y \in Q$. Write $Q y=(Q(1-e) \cap Q y) \oplus Q h$ for some $h \in Q$. Then $y a \neq 0$ implies $y e \neq 0$ and so $h \neq 0$. As $R$ right Utumi implies $Q$ is left intrinsic over $R$, there exists $q \in Q$ with $0 \neq q y \in Q h \cap R$. Now $q y a \neq 0$ and so $0 \neq(q y) a \in Q g \cap R a \subseteq$ 
$J^{\prime} \cap V$. Thus $J^{\prime} \cap V \neq 0$ as desired.

The corollary now follows from Theorem 2.

Corollary 2. Suppose $R$ is a right Utumi ring and $J$ is a left annihilator ideal with a countable-dimensional annihilator base. Then the injective hull of any complement of $r(J)$ is of Type $I_{f}$.

Proof. This follows from the proof of Theorem 2. For in the notation there, $J=Q f \cap R, f Q \cap R$ is a complement of $r_{R}(J)=(1-f) Q \cap R$, and $f Q$ is the injective hull of $f Q \cap R$. As the proof shows, $f Q$ is of Type $I_{f}$.

COROLlaRy 3. If a right Utumi ring $R$ has a faithful right ideal $U$ which is countable dimensional as a left vector space over some division ring $K \subseteq R$, then $Q_{\max }(R)$ is of Type $I_{f}$.

Proof. Let $J=R, V=U$. Since $U$ is faithful, for any nonzero left ideal $J^{\prime} \subseteq J$,

$$
0 \neq U J^{\prime} \subseteq V \cap J^{\prime} .
$$

Hence $J$ is a left annihilator ideal with ${ }_{K} V$ as a CDAB. From $0=r(J)$, we infer $R$ is a complement of $r(J)$ and that $Q_{\max }(R)$ is of Type $I_{f}$ by Corollary 2 .

COROllary 4 . Let $R$ be a prime, right Utumi ring with a nonzero right ideal $U$ which is countable dimensional as a left vector space over some division ring $K \subseteq R$. Then $R$ is right Goldie.

Proof. Immediate from Corollary 3 , since $U$ is faithful in a prime ring and prime, regular, right self-injective rings $Q$ of Type $I_{f}$ are simple Artinian.

REMARKS.

(1) With some hesitation we point out that Corollary 4 provides another characterization of simple Artinian rings, viz. rings which are prime, regular, right Utumi, and contain a countable-dimensional right ideal $\neq 0$. On the other hand, the ring of linear transformations of an infinite-dimensional right vector space is prime, regular, right Utumi and can contain nonzero countable-dimensional left ideals but of course is neither simple or Artinian. (It is of Type $I$, in accordance with Corollary 1.)

(2) In particular, for a right Utumi ring $R$ possessing a left ideal $\neq 0$ with a $\mathrm{CDAB}, Q_{\max }(R)$ can have zero Type $I_{f}$ part (as well as zero Types $I I$ and III parts). Simple examples (such as a direct product of a simple Artinian ring and a Type $I I$ regular right self-injective ring) show that $Q_{\max }(R)$ need not be Type $I$.

(3) It is not true that if a right Utumi ring $R$ contains only countable 
direct sums of nonzero left or right ideals, then $Q_{\max }(R)$ has a nonzero Type $I$ part, e.g. $R$ a simple self-injective ring of Type $I I_{f}$ (see [3, Proposition 5.9]). By Corollary 1, taking $V=R$ and $K=R$ in this situation will not give a $\mathrm{CDAB}{ }_{K} V$ for $R$. Thus the countability requirement for a CDAB ${ }_{K} V$ involves more than simply having only countable direct sums of $K$ submodules of $V$.

A left ideal with a $\mathrm{CDAB}$ over some division ring need not itself be countable dimensional (over any division ring $K$ ) :

\section{EXAMPLE.}

There exists a commutative, regular, self-injective ring $Q$ with no countable-dimensional ideals $(\neq 0)$ but each of its annihilator ideals $J$ has a CDAB over a field. Simply let $Q=Q_{\max }(R)$ where $R$ is a countable Boolean ring without minimal ideals. Let $J=Q f \neq 0$ where $f=f^{2} \in Q$. Because soc $(Q)$ $=0, \oplus_{1}^{\infty} f_{i} Q \leqslant e f Q$ for some nonzero orthogonal idempotents $f_{i}$ and hence by injectivity of $Q_{Q}$

$$
Q f \supseteq \prod_{1}^{\infty} Q f_{i} .
$$

Consequently for any division ring $K \subseteq Q, \operatorname{dim}_{K} Q f$ must be uncountable. However, letting

$$
V=J \cap R \quad \text { and } \quad K=\{0,1\}
$$

we observe that ${ }_{K} V$ is a $\mathrm{CDAB}$ for $J$.

\section{References}

[1] A. W. Chatters and C. R. Hajarnavis: Rings in which every complement right ideal is a direct summand, Quart. J. Math. Oxford 28 (1977), 61-80.

[2] K. R. Goodearl: Ring Theory: Nonsingular Rings and Modules, Pure and Appl. Math., No. 33, Dekker, New York, 1976.

[3] K. R. Goodearl: Von Neumann Regular Rings, Pitman, London, 1979.

[4] J. Hannah: Countability in regular self-injective rings, Quart. J. Math. Oxford 31 (1980), 315-327.

[5] J. HANNAH and K. C. O'Meara: Maximal quotient rings of prime group algebras, Proc. Amer. Math. Soc. 65 (1977), 1-7.

[6] J. LAwrence: A countable self-injective ring is quasi-frobenius, Proc. Amer. Math. Soc., 65 (1977), 217-220.

[7] Y. Utumi : On rings of which any one-sided quotient rings are two-sided, Proc. Amer. Math. Soc. 14 (1963), 141-147. 
John Hannah

Department of Mathematics University College Galway Ireland.

K. C. O'Meara

Department of Mathematics University of Canterbury

Christchurch

New Zealand. 